\title{
REMOÇÃO DE MATÉRIA ORGÂNICA E NITROGÊNIO EM REATOR HÍBRIDO ANAERÓBIO/AERÓBIO/ANÓXICO OPERADO EM BATELADAS SEQUENCIAIS
}

\author{
REMOVAL OF ORGANIC MATTER AND NITROGEN IN ANAEROBIC/ANOXIC HYBRID \\ REACTOR OPERATED ON SEQUENTIAL BATCHES
}

\author{
Heitor Carvalho de Almeida Chagas ${ }^{a}$, Nelia Henriques Calladoa, \\ Marcia Helena Rissato Zamariolli Damianovic ${ }^{b}$
}

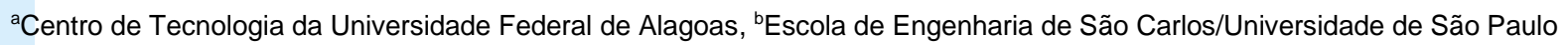
heitorcarvalhoo@hotmail.com, nelia.callado@yahoo.com.br, mzamariollidamianovic@gmail.com

Submissão: 25 de maio de 2020 Aceitação: 05 de outubro de 2020

\section{Resumo}

O crescimento urbano, sem o acompanhamento de sistema de esgotamento sanitário, induz a uma tendência de descentralização de estações de tratamento de efluentes (ETEs), visando à remoção não só de matéria orgânica carbonácea, mas também a remoção/recuperação de nutrientes. $\mathrm{O}$ nitrogênio, além de causar problemas de eutrofização em corpos de água, pode levar à contaminação de águas superficiais e subterrâneas por nitrato, cuja ingestão está associada a efeitos adversos à saúde. Este trabalho apresenta e discute o desempenho de um Reator em Bateladas Sequenciais (RSB), tratando esgoto sanitário, em escala de bancada, operando com ciclos de 24 horas, a fim de remover matéria orgânica e nitrogênio, tendo a fase de reação submetida a períodos anaeróbio, aeróbio e anóxico, com o intuito de avaliar o potencial de incorporação desta tecnologia como opção no tratamento descentralizado de esgotos em empreendimentos residenciais e comerciais. Os resultados mostraram um rápido estabelecimento de eficiência conjunta de remoção de DQO de $70 \%$ e de nitrogênio de $42 \%$, mesmo sem utilizar fonte externa de carbono. Conclui-se que o RSB se apresenta como uma alternativa atraente para aplicação no meio urbano, minimizando os impactos de lançamento dos esgotos no solo, nos sistemas urbanos de drenagem, em corpos de água, bem como atende os padrões ambientais de lançamento.

Palavras-Chave: Reator sequencial em batelada; nitrificação, desnitrificação; esgoto sanitário.

\section{Abstract}

Urban growth without the accompaniment of a sewage system leads to a trend towards decentralization of effluent treatment stations (ETEs), aiming at the removal not only of carbonaceous organic matter, but also the removal / recovery of nutrients. Nitrogen, in addition to causing eutrophication problems in bodies of water, can lead to contamination of surface and groundwater by nitrate, the intake of which is associated with adverse health effects. This work presents and discusses the performance of a Sequential Batch Reactor (RSB), treating sanitary sewage, on a bench scale operating with 24-hour cycles, aiming at the removal of organic matter and nitrogen, with the reaction phase submitted to anaerobic periods, aerobic and anoxic, in order to evaluate the potential of incorporating this technology as an option in the decentralized treatment of sewage in residential and commercial enterprises. The results showed a rapid establishment of joint efficiency in removing COD of $70 \%$ and nitrogen of $42 \%$, even without using an external carbon source. It is concluded that the RSB presents itself as an attractive alternative for application in the urban environment, minimizing the impacts of the discharge of sewers in the soil, in the urban drainage systems, in bodies of water and meeting the environmental standards of release.

Keywords: Sequencing batch reactor; biological nutrient removal; sewage treatment. 


\section{INTRODUÇÃO}

A degradação da qualidade da água, sobretudo nos últimos anos, geralmente, é resultado do lançamento de efluentes nos cursos d'água sem o devido tratamento, principalmente com excesso de nutrientes. No meio urbano, em regiões onde não dispõe de rede coletora, 0 lançamento de esgotos sanitários no solo tem sido reportado como um dos principais responsáveis pela presença de nitrato na água subterrânea (SILVA, 2013). Por outro lado, as questões ambientais têm sido cada vez mais discutidas, o que tem levado à busca $e$ desenvolvimento de novas tecnologias de tratamento das águas residuárias, que possam remover nitrogênio e serem aplicadas em sistemas descentralizados de esgotamento sanitário.

Existem diferentes alternativas para 0 tratamento de esgotos domésticos, algumas complexas e onerosas, outras simples e de baixo custo. Para sistemas descentralizados, principalmente em condomínios residenciais fechados, soluções de baixo custo e de facilidade operacional são fundamentais, pois o sucesso de sua eficiência depende de sua operação, muitas vezes delegadas a operadores de baixa qualificação.

A remoção de matéria carbonácea via processos biológicos aeróbios e anaeróbios é amplamente conhecida. O processo de oxidação por via aeróbia apresenta elevada eficiência (maior que 90\%), mas tem alto custo operacional (consumo de energia) e elevada produção de lodo (maior taxa de crescimento celular), com potencial de oxidação das espécies nitrogenadas presentes no esgoto. Já a degradação por via anaeróbia, embora proporcione menor eficiência (menor que $70 \%$ ), apresenta menor produção de lodo e menor custo operacional do que nos sistemas aeróbios, sem, contudo, influenciar as formas reduzidas constituintes dos esgotos sanitários. Dessa forma, a combinação dos processos anaeróbio e aeróbio traz como vantagem redução no custo de implantação, operação e manutenção devido à menor produção de lodo biológico e à diminuição da demanda de oxigênio (consumo de energia), além da possibilidade de remoção de parcela do nitrogênio, por meio de controle operacional adequado (FOCO; NOUR, 2014).

As alternativas tecnológicas mais usuais para o tratamento de esgotos sanitários baseiamse nos ciclos biogeoquímicos do Carbono e Nitrogênio, sendo que o último se sustenta nos processos de nitrificação e desnitrificação e requer a combinação de ambientes aeróbio/anóxico (ALVES et al., 2018), o que pode dificultar a operação de pequenas ETEs. Nesse contexto, o RSB apresenta-se como uma alternativa atraente, visto que este tipo de reator é capaz de proporcionar diferentes condições ambientais, como anaeróbia, aeróbia e anóxica, em uma mesma unidade, além da operação de separação a partir do controle do fornecimento de oxigênio, descarte e outras operações do ciclo de tratamento que podem ser automatizadas.

A remoção biológica de nitrogênio, por processo convencional, envolve a nitrificação seguida da desnitrificação. A nitrificação é um processo aeróbio em que bactérias autotróficas oxidam a amônia. Já a desnitrificação é um processo anóxico realizado por bactérias heterotróficas que utilizam nitrito e/ou nitrato como aceptor de elétrons, transformando-os em dinitrogênio- $\mathrm{N}_{2}$. Outros gases, como óxido nítrico$\mathrm{NO}$ e óxido nitroso- $\mathrm{N}_{2} \mathrm{O}$, deletérios ao ambiente, podem ser gerados durante $O$ processo $e$ liberados para a atmosfera (PEREIRA et al., 2019). A disponibilidade de doadores de elétrons, como, por exemplo, a matéria orgânica, é necessária para a desnitrificação heterotrófica.

$\mathrm{Na}$ nitrificação, Damianovic et al. (2018) citam que estudos sobre competição entre bactérias autotróficas nitrificantes e heterotróficas degradadoras de matéria carbonácea indicam que a nitrificação é inibida quando a relação $\mathrm{DQO} / \mathrm{N}$ resulta em valores superiores a 4 .

Para desnitrificação, Hu et al. (2014) citam que, em baixa relação entre demanda química de oxigênio (DQO) e concentração de $\mathrm{N}$, o processo biológico de desnitrificação normalmente resulta em baixa eficiência, devido à limitação de carbono disponível.

Os processos de nitrificação e desnitrificação podem ocorrer em conjunto, sendo denominados de Nitrificação e Desnitrificação Simultânea (NDS), quando um reator único tem condições para nitrificação e desnitrificação simultânea, em função da estratificação do biofilme formado (LI et al., 2008).

A busca por tecnologias com maior eficiência de remoção de $\mathrm{N}$ no tratamento de esgotos com baixa carga orgânica tornou-se uma 
demanda urgente (CHEN et al., 2016), e as pesquisas têm buscado aperfeiçoar processos biológicos de modo a promover a remoção conjunta de carbono e nutrientes numa mesma unidade operacional, tornando os sistemas de tratamento ainda mais simples, compactos e flexíveis. Uma solução para esta demanda é a utilização de Reator Sequencial em Batelada (RSB), que desempenha tanto a função de reator biológico como a de decantador, compreendendo ciclos com fases de enchimento, reações biológicas, decantação e repouso. As mudanças na duração da fase de reação dos ciclos, assim como a combinação de fases anaeróbia, aeróbia e anóxica conferem flexibilidade aos RSB, uma vez que o tempo de reação pode ser modificado a depender da carga orgânica. Desta forma, tornase mais viável se obter um efluente dentro dos padrões de lançamento. A utilização do RSB para remoção de matérias carbonácea e nitrogenada tem sido objeto de estudo de vários pesquisadores (CALLADO; FORESTI, 2001; AKIN; UGURLU, 2004; TSUNEDA et al., 2006; LI et al., 2008; XU et al., 2013; LI et al., 2014, SANTOS et al., 2016; BUENO et al., 2019), os quais têm investigado a aplicação desse reator para o tratamento de diferentes substratos, sob diversas configurações operacionais. Neste contexto, o presente estudo objetivou avaliar 0 desempenho de um RSB no que se refere à remoção de matéria orgânica e nitrogênio, tendo a fase de reação submetida a períodos anaeróbio (para remoção de matéria orgânica $e$ amonificação), aeróbio (para nitrificação) e anóxico (para desnitrificação sem fonte externa de carbono). O reator pretende ser avaliado como uma tecnologia para tratamento de esgotos domésticos aplicada a pequenos sistemas descentralizados, no meio urbano, visando a estudar o seu potencial de reduzir o aporte de carga orgânica e de nitrogênio proveniente dos esgotos sanitários, na rede de drenagem e nos cursos de água urbanos.

\section{MÉTODOS}

\section{Configuração e planejamento experimental}

A pesquisa foi desenvolvida utilizando um Reator Sequencial em Batelada no formato cilíndrico, em escala de bancada, construído em acrílico, com volume útil de $8 \mathrm{~L}$ (6 litros de substrato e 2 litros de lodo) e altura $53 \mathrm{~cm}$. A
Figura 1 mostra a instalação experimental e seus principais equipamentos de controle.

O reator foi operado por 72 dias, à temperatura ambiente, com tempo do ciclo de $24 \mathrm{~h}$, que resultava em uma batelada por dia, em quatro fases distintas, como apresentado na Tabela 1.

O ciclo de $24 \mathrm{~h}$ foi utilizado na operação dos RSB com o intuito de não limitar o desempenho do reator pelo tempo de reação do ciclo, visto que - mesmo estava iniciando sua operação com lodos de inóculo, os quais ainda não estavam adaptados nem ao substrato sintético e nem às condições operacionais do reator, e os processos de nitrificação e desnitrificação ainda precisavam ser estabelecidos. A configuração temporal de cada fase do ciclo foi baseada nos dados da literatura (CALLADO; FORESTI, 2001; PICKBRENNER, 2002; BUENO et al, 2019). Após o estabelecimento do estado estacionário dinâmico do reator, com respeito ao estabelecimento da remoção de matéria orgânica e da nitrificação e desnitrificação, foi realizado um perfil temporal de análises. Amostras foram retiradas a cada 2 horas, durante um ciclo completo de operação, para se verificar a evolução, ao longo do ciclo, dos parâmetros de interesse nos processos de remoção de nitrogênio e matéria orgânica.

$O$ enchimento do reator era feito por gravidade, abrindo-se manualmente o registro do reservatório de alimentação. Após o enchimento, iniciava-se a fase de reação, compreendendo as condições anaeróbia, aeróbia e anóxica. $\mathrm{Na}$ fase seguinte, ocorria a separação da massa líquida da sólida em suspensão por meio da sedimentação e, na última fase, o descarte do sobrenadante, por gravidade, abrindo-se o registro de descarte do efluente tratado localizado no ponto acima da manta de lodo sedimentada.

$\mathrm{Na}$ condição anaeróbia da fase de reação, a bomba peristáltica era acionada, promovendo a recirculação do substrato do topo para o fundo do reator, proporcionando, dessa forma, o contato permanente com a biomassa sedimentada no fundo do reator, com vazão de recirculação de $0,12 \mathrm{~L} / \mathrm{min}$. Na condição seguinte, aeróbia, a bomba de recirculação era desligada e o aerador era acionado, promovendo a suspensão da biomassa, sua mistura com o substrato e fornecendo oxigênio dissolvido ao meio. $\mathrm{Na}$ condição anóxica, o aerador era desligado, o lodo 
sedimentava e a bomba de recirculação acionada novamente de forma semelhante à fase anaeróbia. O controle operacional do sistema, no que se refere ao controle liga/desliga da bomba de recirculação e liga/desliga do aerador, era executado por meio de temporizadores previamente programados, cuja programação permaneceu durante todo o experimento.

Figura 1 - Esquema da instalação experimental

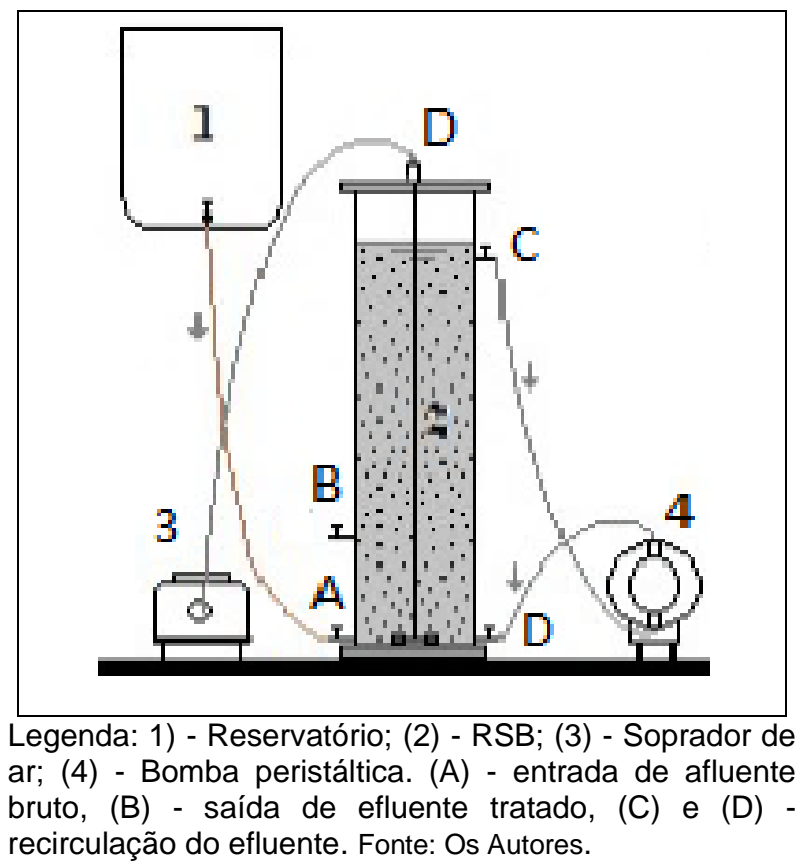

Tabela 1 - Fases do ciclo operacional do RSB

\begin{tabular}{lcccc}
\multicolumn{2}{c}{ Fase do ciclo } & Tempo & Recirculação & Aeração \\
Enchimento & & $0,25 \mathrm{~h}$ & Desligada & Desligada \\
\hline \multirow{2}{*}{ Reação } & Anaeróbia & $8,0 \mathrm{~h}$ & Ligada & Desligada \\
& Aeróbia & $7,0 \mathrm{~h}$ & Desligada & Ligada \\
& Anóxica & $7,0 \mathrm{~h}$ & Ligada & Desligada \\
\hline Sedimentação & & $1,5 \mathrm{~h}$ & Desligada & Desligada \\
\hline Descarte & & $0,25 \mathrm{~h}$ & Desligada & Desligada \\
\hline
\end{tabular}

Fonte: Os Autores.

\section{Substrato e lodo de inóculo}

O reator era alimentado todos os dias com $6 \mathrm{~L}$ de substrato sintético, preparado diariamente, segundo adaptação da composição descrita por Torres (1992). A composição do substrato sintético assemelhava-se ao esgoto sanitário real com DQO em torno de $850 \mathrm{mg} / \mathrm{L}$ e sua escolha sustentou-se, principalmente, na redução do risco de contaminação biológica e na facilidade de preparo. A composição do substrato adaptada de Torres (1992) está apresentada na Tabela 2.

Além dos compostos apresentados, adicionou-se à preparação do substrato sintético,
200mg/L de Bicarbonato de Sódio $\left(\mathrm{NaHCO}_{3}\right)$, com o objetivo de manter $\mathrm{o} \mathrm{pH}$ próximo à neutralidade. $\mathrm{O}$ esgoto também foi suplementado com ureia $\left(\left(\mathrm{NH}_{2}\right)_{2} \mathrm{CO}\right)$, a fim de atingir um valor de nitrogênio afluente típico de esgoto sanitário real forte, em torno de $54 \mathrm{mg} . \mathrm{N} / \mathrm{L}$.

Para inocular o RSB, foram utilizados $2,0 \mathrm{~L}$ de lodo, sendo $50 \%$ (em volume) proveniente de um sistema de Lodos Ativados aeróbio e $50 \%$ de lodo anaeróbio de reator UASB, ambos de Estações de Tratamento de Esgoto doméstico. A Tabela 3 apresenta a caracterização dos lodos de inóculo. 
Tabela 2 - Composição do esgoto sanitário sintético

\begin{tabular}{cccc}
\hline Composto & DQO & Fonte & Observações \\
\hline Proteínas & $50 \%$ & $2 \mathrm{~mL} / \mathrm{L}$ de Fígado bovino & Solução estoque com DQO conhecida \\
\hline Carboidratos & $40 \%$ & $\begin{array}{c}53 \mathrm{mg} / \mathrm{L} \text { de Sacarose } \\
165 \mathrm{mg} / \mathrm{L} \text { de Amido Comercial } \\
60 \mathrm{mg} / \mathrm{L} \text { de Celulose }\end{array}$ & $\begin{array}{c}\text { Previamente pesada } \\
\text { Previamente pesado } \\
\text { Papel higiênico picotado e pesado }\end{array}$ \\
\hline Lipídios & $10 \%$ & $0,08 \mathrm{~mL} / \mathrm{L}$ de Óleo de soja, & $\begin{array}{c}\text { Emulsionado com } 3 \text { gotas de } \\
\text { detergente/L } / \mathrm{L} / \mathrm{L}\end{array}$ \\
\hline Sais minerais & - & $\begin{array}{r}5 \mathrm{~mL} / \mathrm{L} \text { de solução estoque, } \\
\text { previamente preparada com: }\end{array}$ & $\begin{array}{c}\mathrm{NaCl}-50,0 \mathrm{~g} / \mathrm{L} \\
\mathrm{MgCl}_{2} .6 \mathrm{H}_{2} \mathrm{O}-1,4 \mathrm{~g} / \mathrm{L} \\
\mathrm{CaCl}_{2}-0,9 \mathrm{~g} / \mathrm{L}\end{array}$ \\
\hline
\end{tabular}

Fonte: Adaptada de Torres, 1992.

Tabela 3 - Caracterização dos lodos de inóculo do RSB

\begin{tabular}{ccc}
\hline Parâmetro & Lodo Aeróbio & Lodo Anaeróbio \\
\hline $\mathbf{p H}$ & 7,0 & 6,7 \\
ST (mg/L) & 10.440 & 15.450 \\
STV/ST & 0,78 & 0,49
\end{tabular}

Legenda: ST - Sólidos Totais; STV - Sólidos Totais Voláteis. Fonte: Os Autores.

\section{Determinações analíticas}

Para o monitoramento dos sistemas, foram analisados os parâmetros apresentados na Tabela 4, com os respectivos métodos de determinação e frequência de análise.

Para análise de alcalinidade total (AT), intermediária $(\mathrm{Al})$ e bicarbonato $(\mathrm{AP})$, utilizou-se $\mathrm{o}$ método titulométrico modificado por Ripley, Boyle e Converse (1986). As análises de nitrato e nitrito foram realizadas segundo Mackereth et al. (1978), dos ácidos volateis (AV), segundo Dillalo e Albertson (1961) e as demais análises, de acordo com técnicas do Standard Methods for the Examination of Water And Wastewater (APHA, AWWA, WPCF, 2017). Tais análises realizaramse no Laboratório de Saneamento Ambiental do Centro de Tecnologia da Universidade Federal de Alagoas (LSA/CTEC/UFAL).

\section{RESULTADOS E DISCUSSÃO}

O reator funcionou 72 dias (72 ciclos) à temperatura ambiente, na faixa mesofílica, variando de $27,3^{\circ} \mathrm{C} \pm 1,61$ a $33,5{ }^{\circ} \mathrm{C} \pm 1,64$ ao longo do tempo. Os valores médios e desvio padrão dos parâmetros avaliados constam na Tabela 5.
Como o NTK no afluente encontrava-se praticamente todo na forma orgânica, a eficiência dos processos de amonificação, nitrificação e desnitrificação foi avaliada de forma global pela remoção de nitrogênio $\mathrm{N}$-total $\left(\mathrm{N}-\mathrm{org}+\mathrm{N}-\mathrm{NH}_{4}{ }^{+}+\right.$ $\left.\mathrm{N}-\mathrm{NO}_{2}{ }^{-}+\mathrm{N}-\mathrm{NO}_{3}{ }^{-}\right)$, desconsiderando-se $\mathrm{O}$ consumo por assimilação e as perdas de amônia por volatilização. Desta forma, o balanço médio de nitrogênio, apresentado na Tabela 6 , mostra que o RSB removeu apenas cerca de 19,97mg.N/L, o que resultou numa eficiência média de $36,7 \%$. No final da operação, essa eficiência alcançou o valor de $42 \%$, quando o $\mathrm{N}$ total afluente era de $54 \mathrm{mg} . \mathrm{N} / \mathrm{L}$ e a do efluente de $32 \mathrm{mg} . \mathrm{N} / \mathrm{L}$, com $\mathrm{N}$-amon de $17 \mathrm{mg} / \mathrm{L}$ atendendo aos padrões de lançamento da Resolução 430/2011 do CONAMA (Brasil, 2011).

A especiação das formas de nitrogênio presentes no efluente do reator mostrou que os processos de amonificação, nitrificação e desnitrificação não eram completos, pois o efluente ainda continha nitrogênio na forma amoniacal e de nitrato. Para o período, foram registrados valores médios de efluentes de 10,26 $\pm 5,13 \mathrm{mg} / \mathrm{L}, 6,44 \pm 3,16 \mathrm{mg} / \mathrm{L}$ e $0,02 \pm 0,01 \mathrm{mg} / \mathrm{L}$ para o $\mathrm{N}$-amoniacal, $\mathrm{N}$-nitrato e $\mathrm{N}$-nitrito, respectivamente, como apresentado na Tabela 6 . 
Tabela 4 - Parâmetros analisados, métodos e frequência de análises

\begin{tabular}{|c|c|c|c|}
\hline Parâmetro & Método & Frequência & $N^{\circ}$ de análises \\
\hline $\mathrm{pH}$ & Potenciométrico & Diário & 72 \\
\hline $\begin{array}{l}\text { Alcalinidade } \\
\left(\mathrm{mg} \cdot \mathrm{CaCO}_{3} / \mathrm{L}\right)\end{array}$ & Titulométrico - Ripley et al., 1986 & 2x Semana & $\begin{array}{c}20 \text { em } \\
\text { duplicatas }\end{array}$ \\
\hline Ácidos Voláteis (mg/L) & Titulométrico - Dilalo e Albertson, 1961 & 2x Semana & $\begin{array}{c}20 \text { em } \\
\text { duplicatas }\end{array}$ \\
\hline $\mathrm{DQO}(\mathrm{mg} / \mathrm{L})$ & Espectrofotométrico - 5220 C & 2x Semana & $\begin{array}{c}20 \text { em } \\
\text { duplicatas }\end{array}$ \\
\hline ST, SF e SV (mg/L) & Gravimétrico - 2540 D, 2540 E & 2x Semana & $\begin{array}{c}20 \text { em } \\
\text { duplicatas }\end{array}$ \\
\hline N-NTK (mg/L) & Titulométrico - 4500 - N-org C & 2x Semana & $\begin{array}{c}20 \text { em } \\
\text { duplicatas }\end{array}$ \\
\hline $\begin{array}{c}\text { Amônio: } \mathrm{N}-\mathrm{NH}_{4}+(\mathrm{mg} \\
\mathrm{N} / \mathrm{L})\end{array}$ & Titulométrico - $4500-\mathrm{NH}_{3}$ & 2x Semana & $\begin{array}{l}20 \text { em } \\
\text { duplicatas }\end{array}$ \\
\hline Nitrito: $\mathrm{N}-\mathrm{NO}_{2}^{-}$(mg N/L) & $\begin{array}{l}\text { Espectrofotométrico-Mackereth et al. } \\
1978\end{array}$ & 2x Semana & $\begin{array}{l}20 \text { em } \\
\text { duplicatas }\end{array}$ \\
\hline Nitrato: $\mathrm{N}^{-\mathrm{NO}_{3}}{ }^{-}(\mathrm{mg} \mathrm{N} / \mathrm{L})$ & $\begin{array}{l}\text { Espectrofotométrico-Mackereth et al. } \\
1978\end{array}$ & 2x Semana & $\begin{array}{c}20 \text { em } \\
\text { duplicatas }\end{array}$ \\
\hline
\end{tabular}

Tabela 5 - Valores médios dos parâmetros físico-químicos avaliados na operação do RSB

\begin{tabular}{|c|c|c|c|c|}
\hline \multirow{2}{*}{ Parâmetros } & \multicolumn{2}{|c|}{ Afluente } & \multicolumn{2}{|c|}{ Efluente } \\
\hline & Média & Desvio padrão & Média & Desvio Padrão \\
\hline $\mathrm{pH}$ & 7,53 & $\pm 0,40$ & 7,19 & $\pm 0,43$ \\
\hline AT, mg/L & 96,42 & $\pm 25,25$ & 220,46 & $\pm 62,44$ \\
\hline Al, mg/L & 6,56 & $\pm 2,89$ & 21,15 & $\pm 14,67$ \\
\hline $\mathrm{AP}, \mathrm{mg} / \mathrm{L}$ & 89,86 & $\pm 24,47$ & 199,31 & $\pm 62,95$ \\
\hline $\mathrm{AV}, \mathrm{mg} / \mathrm{L}$ & 23,72 & $\pm 5,13$ & 42,76 & $\pm 27,80$ \\
\hline DQO, mg/L & 843,65 & $\pm 103,20$ & 373,64 & $\pm 146,75$ \\
\hline NTK, mg/L & 54,00 & $\pm 5,50$ & 28,00 & $\pm 3,65$ \\
\hline $\mathrm{N}-\mathrm{NH}_{4+}, \mathrm{mg} / \mathrm{L}$ & 0,70 & $\pm 0,35$ & 10,26 & $\pm 5,13$ \\
\hline $\mathrm{N}-\mathrm{NO}_{2-}, \mathrm{mg} / \mathrm{L}$ & - & - & 0,02 & $\pm 0,01$ \\
\hline $\mathrm{N}-\mathrm{NO}_{3^{-}}, \mathrm{mg} / \mathrm{L}$ & - & - & 6,44 & $\pm 3,16$ \\
\hline $\mathrm{ST}, \mathrm{mg} / \mathrm{L}$ & 1084,00 & $\pm 501,51$ & 445,0 & $\pm 161,31$ \\
\hline $\mathrm{SV}, \mathrm{mg} / \mathrm{L}$ & 984,14 & $\pm 489,11$ & 263,50 & $\pm 116,22$ \\
\hline $\mathrm{SF}, \mathrm{mg} / \mathrm{L}$ & 199,86 & $\pm 83,60$ & 181,50 & $\pm 74,11$ \\
\hline
\end{tabular}

Fonte: Os Autores.

Tabela 6 - Balanço de nitrogênio pelas médias observadas

\begin{tabular}{ccc}
\hline Forma de $\mathbf{N}$ & Afluente & Efluente \\
\hline $\mathrm{N}-\mathrm{org}, \mathrm{mg} / \mathrm{L}$ & $53,73 \pm 5,11$ & $17,74 \pm 3,84$ \\
$\mathrm{~N}-\mathrm{NH}_{4+}, \mathrm{mg} / \mathrm{L}$ & $0,7 \pm 0,35$ & $10,26 \pm 5,13$ \\
$\mathrm{~N}-\mathrm{NO}_{2}-, \mathrm{mg} / \mathrm{L}$ & - & $0,02 \pm 0,01$ \\
$\mathrm{~N}-\mathrm{NO}_{3-}, \mathrm{mg} / \mathrm{L}$ & - & $6,44 \pm 3,16$ \\
\hline $\mathrm{N}$-total & $54,43 \pm 5,50$ & $34,46 \pm 6,31$ \\
\hline
\end{tabular}

Fonte: Os Autores. 
Considerando que o valor médio do NTK no efluente tratado era de $28,0 \mathrm{mg} . \mathrm{N} / \mathrm{L}$, e que a concentração média de $\mathrm{N}$-amoniacal era de 10,26mg.N/L, existiam ainda 17,74mg.N/L (28,0 $10,26=17,74)$ na forma orgânica que não estavam disponíveis para nitrificação, e que $36,69 \mathrm{mg} . \mathrm{N} / \mathrm{L}(54,43-17,74=36,69)$ foram amonificados, ou seja, a eficiência média de amonificação foi de $67,4 \%$.

$\mathrm{O}$ balanço entre a concentração de $\mathrm{N}$ amoniacal disponível (36,69mg.N/L) e a concentração média de $\mathrm{N}$-amoniacal remanescente no efluente tratado de 10,26mg.N/L evidencia o estabelecimento do processo de nitrificação, no qual tem-se que $26,43 \mathrm{mg} . \mathrm{N} / \mathrm{L}(36,69-10,26=26,43)$ foram nitrificados, resultando numa eficiência média de nitrificação de $72,03 \%$.

Por sua vez, o estabelecimento do processo de desnitrificação ficou demonstrado pelo balanço do $\mathrm{N}$-nitrato disponível para desnitrificação $(26,43 \mathrm{mg} . \mathrm{N} / \mathrm{L})$, ou seja, o N-nitrato $(6,44 \quad \mathrm{mg} . \mathrm{N} / \mathrm{L})$ e $0 \quad \mathrm{~N}$-nitrito (0,02mg.N/L) presentes no efluente tratado, quando se verifica que $19,97 \mathrm{mg} . \mathrm{N} / \mathrm{L}$ foram desnitrificados, indicando uma eficiência de desnitrificação de $75,6 \%$. A Figura 2 mostra as diferentes formas de nitrogênio presentes no efluente tratado.

Figura 2 - Concentrações do N-nitrito, N-nitrato e N-amoniacal

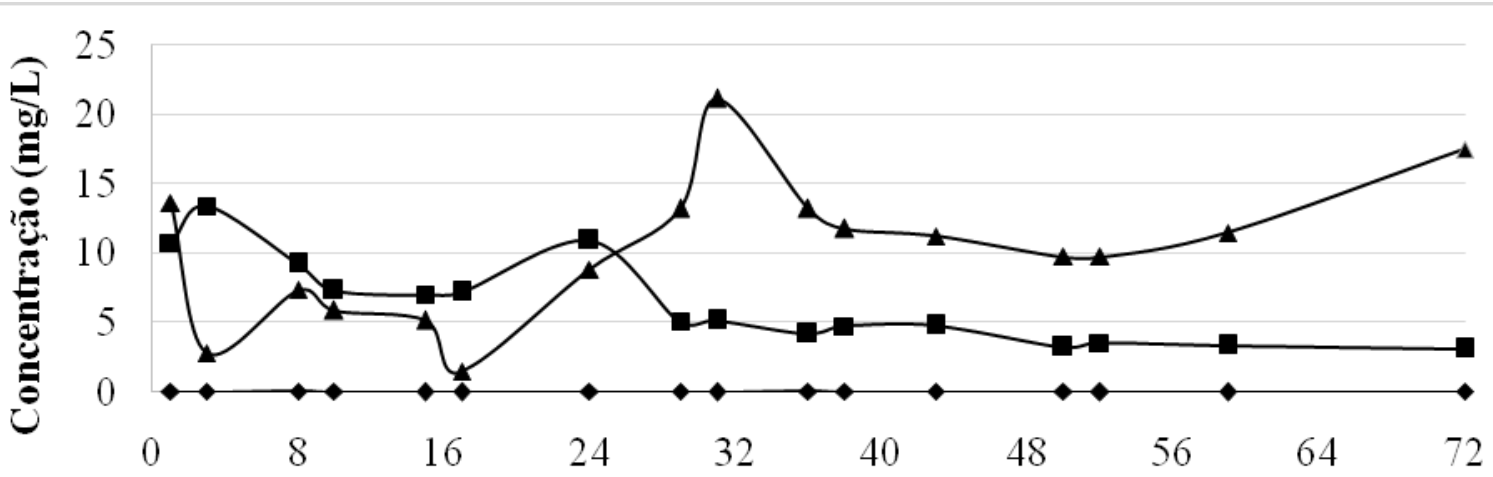

Tempo de operação (dias)

$\rightarrow-N$-nitrato $\rightarrow-N$-nitrito $\rightarrow$ N-amoniacal

Fonte: Os Autores.

A partir da Figura 2, observa-se que até 0 $24^{\circ}$ ciclo (24ํำ dia de operação), a concentração de nitrato no efluente era maior que a do $\mathrm{N}$ amoniacal, invertendo a partir daí. A Tabela 7 mostra os valores médios da concentração de $\mathrm{N}$ amoniacal e N-nitrato, antes e depois do $24^{\circ}$ dia.

Esses resultados podem indicar que, a partir do $24^{\circ}$ ciclo, os processos de amonificação e de desnitrificação estavam começando a se consolidar, mas que o processo de nitrificação estava com alguma limitação, visto que o $\mathrm{N}$ amoniacal disponível começou a se acumular. Aparentemente, esse balanço de massa de nitrogênio, embora simplista, mostra que não houve acúmulo de nitritos por qualquer processo de inibição do processo de nitratação, mas que a limitação poderia estar na etapa de nitritação, que é a que demanda maior disponibilidade de oxigênio disponível. Foi verificado que a concentração de OD, embora tivesse sido planejada para uma concentração superior a $2 \mathrm{mg} . \mathrm{O}_{2} / \mathrm{L}$, o soprador de ar só estava conseguindo inserir $1,13 \mathrm{mg} . \mathrm{O}_{2} / \mathrm{L}$ na fase aeróbia, ou seja, não estava na faixa desejada de 2,0 a 4,0mg.O $/$ L (METCALF; EDDY, 2016) para a nitrificação.

A capacidade de desnitrificação está relacionada com a quantidade de substrato facilmente biodegradável (relação DQO/nitrogênio) na fase anóxica. Neste estudo, a matéria orgânica disponível na fase anóxica era a DQO remanescente das fases anaeróbia e aeróbia. Assim, considerando o valor da DQO remanescente no efluente final do RSB, tendo em 
vista o valor médio de $\mathrm{N}$-amoniacal nitrificado, foi calculada a relação $\mathrm{DQO} / \mathrm{N}$, apresentada na Tabela 8.

A razão $\mathrm{DQO} / \mathrm{N}$ observada foi de 14,13 , para uma eficiência de desnitrificação de $75,6 \%$. Este valor está maior do que os valores encontrados na literatura. Como exemplos, podem ser citados Chiu et al. (2007), que obtiveram $98,7 \%$ de eficiência de remoção de nitrogênio total com relação $\mathrm{DQO} / \mathrm{N}$ de 11,1 ,
Fontenot et al. (2007), eficiência maior que $91 \%$ com relação $\mathrm{DQO} / \mathrm{N}$ variando de 5 a 10 , e Meng et al. (2008), 73\% de eficiência de nitrogênio total com relação $\mathrm{DQO} / \mathrm{N}$ de 9,59. Vale ressaltar que, embora o tempo da fase de reação do ciclo do RSB fosse menor que o aplicado neste trabalho, essa relação citada na literatura é de uma DQO facilmente degradável, ao contrário da calculada aqui, em que a matéria orgânica foi remanescente de processos biológicos anaeróbio e aeróbio.

Tabela 7 - Valores médios das formas nitrogenadas observadas antes e depois do $24^{\circ}$ ciclo do RSB

\begin{tabular}{|c|c|c|}
\hline Forma do $\mathrm{N}$ & Até o 24ํㅜ ciclo & Depois do $24^{\circ}$ ciclo \\
\hline $\mathrm{N}$-amoniacal (mg.N/L) & $6,4 \pm 4,3$ & $13,1 \pm 3,8$ \\
\hline N-nitrato (mg.N/L) & $9,4 \pm 2,4$ & $4,1 \pm 0,7$ \\
\hline $\mathrm{N}$-nitrito (mg.N/L) & $0,02 \pm 0,01$ & $0,02 \pm 0,01$ \\
\hline
\end{tabular}

Fonte: Os Autores.

Tabela 8 - Relação DQO/N observada na operação do RSB

\begin{tabular}{ccc}
\hline DQO remanescente $(\mathbf{m g} / \mathbf{L})$ & $\mathbf{N}$-amoniacal $(\mathbf{m g} / \mathbf{L})$ & Relação DQO/N \\
\hline 373,6 & 26,43 & 14,13
\end{tabular}

Fonte: Os Autores.

Para melhor compreensão do comportamento dos principais parâmetros envolvidos nos processos de remoção de nitrogênio, foi realizado, no final da operação do RSB (ciclo 72), o perfil temporal apresentado na Figura 3.

A partir do gráfico da Figura 3, observa-se que o processo de amonificação foi predominante na fase I (anaeróbia), ocorrendo nas 2 primeiras horas, permanecendo basicamente constante até o final da fase I, mas voltando a ser estimulado na fase aeróbia. Também não se verifica na fase aeróbia qualquer ponto indicativo do final do processo de nitrificação, como era esperado. A expectativa era de que a nitrificação ocorresse na fase aeróbia e a desnitrificação na fase anóxica, mas os resultados indicaram desnitrificação na fase aerada. O comportamento observado no perfil temporal mostra o decréscimo progressivo do NTK nas fases aeróbia e anóxica, a manutenção do $\mathrm{N}$-amoniacal na faixa de 18 mg.N/L, e um equilíbrio no nitrato na faixa de $4 \mathrm{mg} . \mathrm{N} / \mathrm{L}$, sugerindo que nas fases aeróbias e anóxica estava ocorrendo o processo de nitrificação e desnitrificação simultânea (NDS), visto que a concentração de OD estava na faixa ótima de OD para remoção de nitrogênio por NDS de 0,1 a 1,5mg. $\mathrm{O}_{2} / \mathrm{L}$ (ZOPPAS et al., 2016).

A nitrificação depende da temperatura e do $\mathrm{pH}$, já que esses parâmetros determinam o equilíbrio entre as formas ionizadas e não ionizadas dos substratos. Segundo Metcalf e Eddy (2016), para valores de pH entre 10 e 12, o nitrogênio amoniacal encontra-se predominantemente na forma de amônia livre $\left(\mathrm{NH}_{3}\right)$, o que inibe as bactérias oxidantes de amônia. Como o pH do RSB foi mantido em torno de 7,0 e a temperatura do meio líquido na faixa de $27^{\circ} \mathrm{C}$ a $35^{\circ} \mathrm{C}$, praticamente todo nitrogênio amoniacal apresentava-se na forma ionizada ( $\mathrm{N}$ $\mathrm{NH}_{4}{ }^{+}$), e a amônia livre $\left(\mathrm{N}-\mathrm{NH}_{3}\right)$ presente não causava inibição das bactérias oxidadoras de amônia.

$\mathrm{O} \mathrm{pH}$ observado tanto para o esgoto afluente quanto $\mathrm{o}$ esgoto efluente apresentou valores médios próximos a neutralidade, 7,53 \pm 0,40 e 7,19 $\pm 0,43$ (Tabela 2), respectivamente. Esses valores verificados permaneceram dentro da faixa recomendada por Metcalf e Eddy (2016) para a ocorrência de nitrificação $(\mathrm{pH}$ entre 7,2 a 9,0 ), desnitrificação ( $\mathrm{pH}$ de 6,5 a 7,5), bem como para remoção de material carbonáceo $(\mathrm{pH}$ entre 
6,0 a 9,0). Esses valores próximos da neutralidade podem ter contribuído para garantir condições de $\mathrm{pH}$ favoráveis ao crescimento e adaptação de microrganismos responsáveis pela nitrificação, o que foi um fator importante nesse período de adaptação do reator.

A estabilidade do $\mathrm{pH}$ está relacionada com a alcalinidade presente no efluente, cujo valor médio no esgoto afluente foi de $89,86 \pm$ $24,47 \mathrm{mgCaCO}_{3} / \mathrm{L}$, enquanto $\mathrm{o}$ esgoto efluente

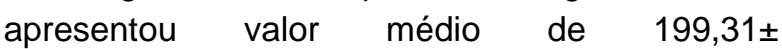
$62,95 \mathrm{mgCaCO}_{3} / \mathrm{L}$. A literatura recomenda um valor de $70 \mathrm{mg} \cdot \mathrm{CaCO}_{3} / \mathrm{L}$, necessário para manutenção da biomassa estável em reator de lodo ativado tratando esgoto sanitário.

Figura 3 - Perfil temporal das formas de nitrogênio durante o último ciclo operacional

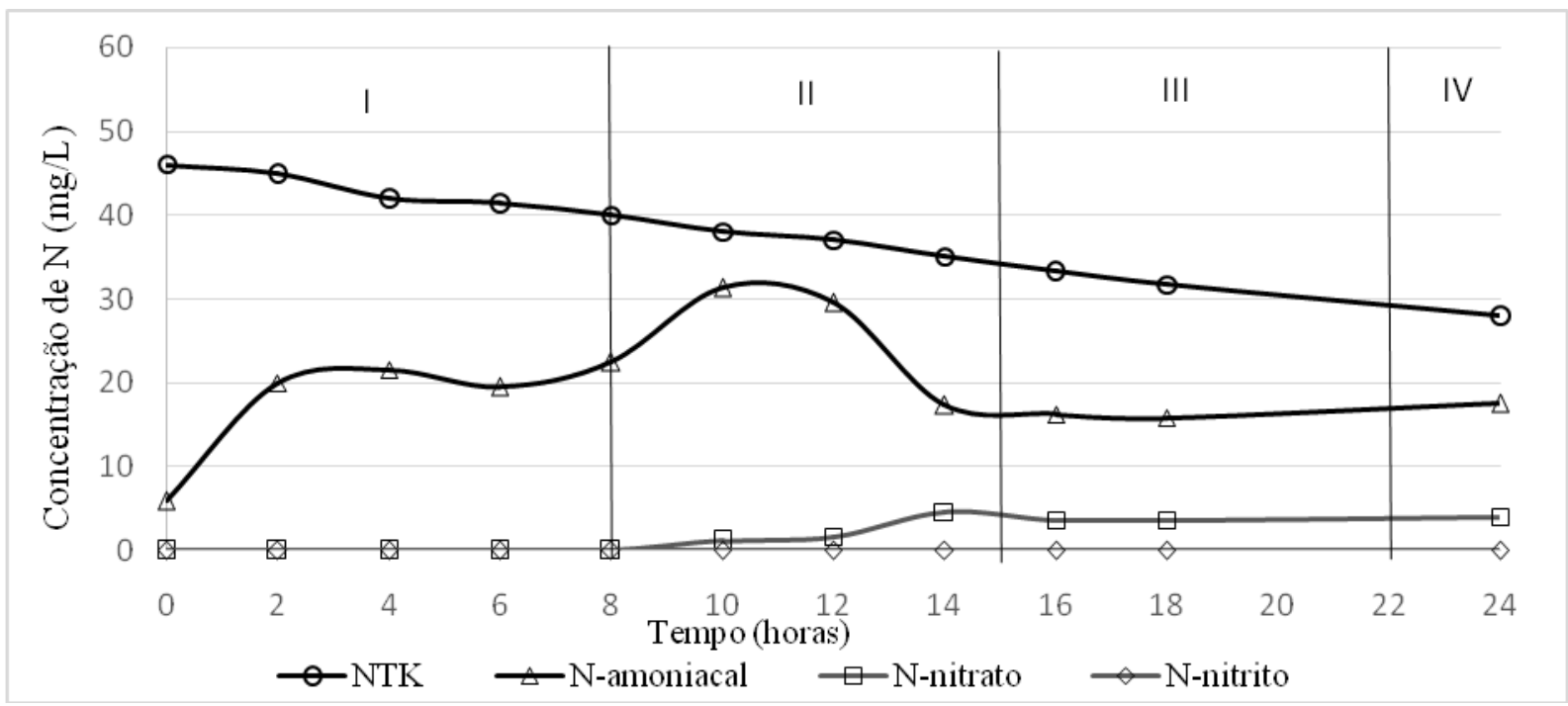

Legenda: I - Fase anaeróbia, II - Fase aeróbia, III - Fase anóxica, IV - Sedimentação/Descarte Fonte: Os Autores.

Analisando os valores de alcalinidade apresentados, verifica-se que houve um aumento de alcalinidade no RSB. A expectativa é de que esse aumento de alcalinidade no RSB deva-se à maior geração de alcalinidade da remoção carbonácea, pelos processos de amonificação e metanogênese na condição anaeróbia, e da desnitrificação nas condições subsequentes, em relação ao consumo de alcalinidade do processo de nitrificação. O comportamento da alcalinidade total no período estudado pode ser observado no gráfico da Figura 4.

Estequiometricamente 0 processo de amonificação gera de $3,57 \mathrm{mg}$, em função de $\mathrm{CaCO}_{3}$, por $\mathrm{mg}$ de nitrogênio amonificado, enquanto o processo de nitrificação consome $7,14 \mathrm{mg}$ de alcalinidade por $\mathrm{mg}$ de nitrogênio oxidado, e o de desnitrificação repõe $3,57 \mathrm{mg}$ de alcalinidade por $\mathrm{mg}$ de nitrato reduzido (CALLADO; FORESTI, 2001). Levando-se em consideração os valores médios de nitrogênio amonificado, oxidado (na nitrificação) e reduzido (na desnitrificação), foi feito o balanço teórico de alcalinidade no RSB apresentado na Tabela 9.

Por meio do balanço estequiométrico dos processos de amonificação, nitrificação e desnitrificação no RSB, verificou-se que a tendência era de aumento médio de $14 \mathrm{mg} . \mathrm{CaCO}_{3} / \mathrm{L}$ de alcalinidade. No entanto, o aumento médio de alcalinidade observado foi de $124 \mathrm{mg} . \mathrm{CaCO}_{3} / \mathrm{L}$. Essa diferença deve-se, certamente, a outros processos que ocorreram no reator como a metanogênese ou, até mesmo, a volatilização de $\mathrm{CO}_{2}$.

Os dados de alcalinidade média do substrato sintético era de $96 \mathrm{mg} \cdot \mathrm{CaCO}_{3} / \mathrm{L}$, e na condição anaeróbia, no início do ciclo de reação, eram, teoricamente, gerados $131 \mathrm{mg} \cdot \mathrm{CaCO}_{3} / \mathrm{L}$. Assim, a alcalinidade média disponível para

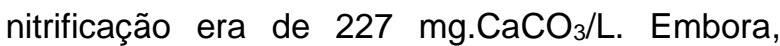
para nitrificar todo nitrogênio amonificado (37mg. $\left.\mathrm{CaCO}_{3} / \mathrm{L}\right), \quad$ fossem necessários 
262mg. $\mathrm{CaCO}_{3} / \mathrm{L}$ (superior a alcalinidade teórica disponível), os dados de alcalinidade remanescente no RSB, superiores a do afluente, indicam a existência de outros processos que, também, contribuíam para geração de alcalinidade, e que a alcalinidade não deve ter sido fator limitante para a nitrificação incompleta verificada no RSB.

Figura 4 - Comportamento da Alcalinidade Total

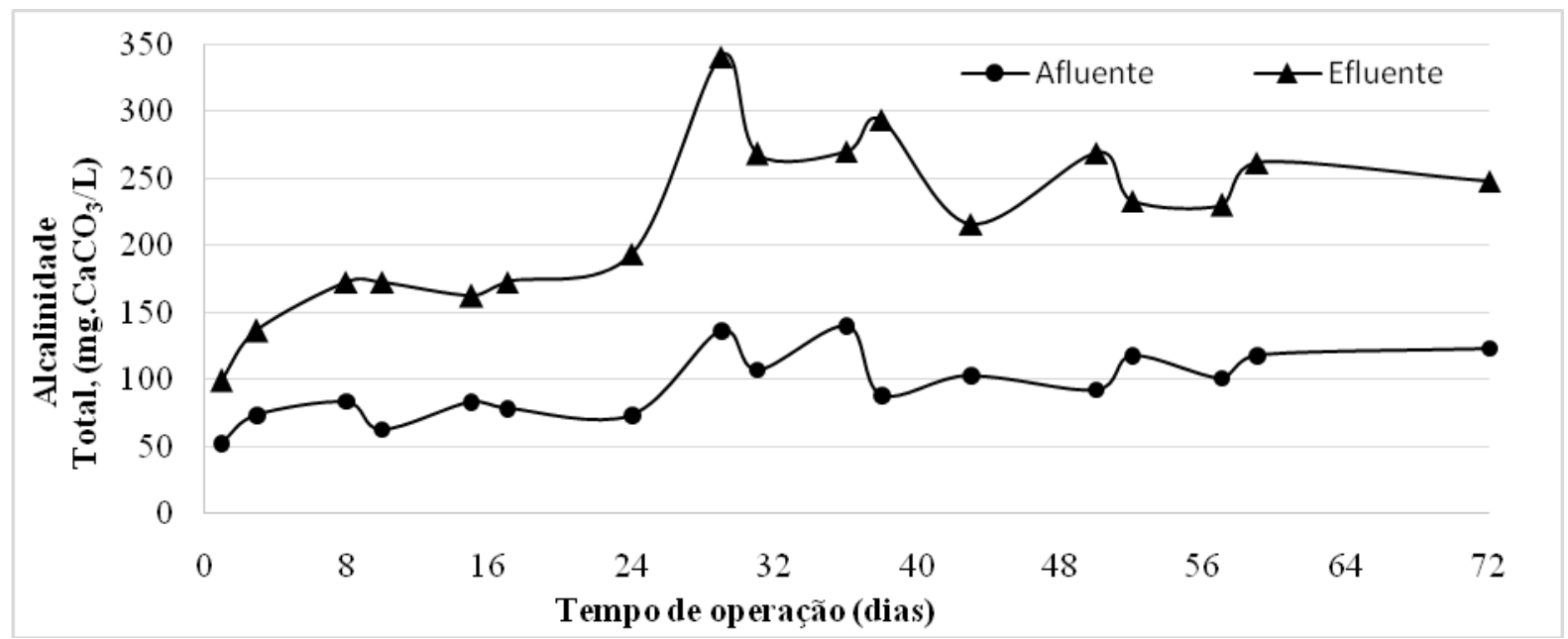

Fonte: Os Autores.

Tabela 9 - Balanço teórico de alcalinidade na remoção de nitrogênio para os valores médios obtidos

\begin{tabular}{|c|c|c|}
\hline Processo & Nitrogênio (mg.N/L) & Alcalinidade $\left(\mathrm{mg} \cdot \mathrm{CaCO}_{3} / \mathrm{L}\right)$ \\
\hline Amonificação & 36,69 & $+130,98$ \\
\hline Nitrificação & 26,43 & $-188,71$ \\
\hline Desnitrificação & 19,97 & $+71,29$ \\
\hline
\end{tabular}

Fonte: Os Autores.

A relação entre a alcalinidade intermediária (devida a ácidos fracos) e alcalinidade parcial (devido a bicarbonatos) - Al/AP, utilizada em reatores anaeróbios para avaliar a produção e o consumo de ácidos, foi de 0,11 no efluente do RSB. Os ácidos gerados na condição anaeróbia inicial, certamente, foram utilizados nas condições (aeróbia e anóxica) subsequentes. De acordo com Gonzales et al. (2015), os ácidos graxos voláteis (AGV's) gerados no processo anaeróbio podem ser utilizados na desnitrificação, uma vez que são facilmente metabolizados por bactérias heterotróficas desnitrificantes.

A DQO afluente apresentou como valor médio $843 \pm 103 \mathrm{mg} / \mathrm{L}$, enquanto o valor médio de DQO efluente foi de $374 \pm 147 \mathrm{mg} / \mathrm{L}$. Foi possível observar certa instabilidade na eficiência de remoção de DQO, sobretudo nas três primeiras semanas, o que já era esperado devido à necessidade de adaptação dos microrganismos ao substrato sintético e às diferentes condições operacionais (anaeróbio/aeróbio/anóxico) durante o período de reação da batelada. Os valores máximo e mínimo para $D Q O$ (ef) para o período foram 645 e $157 \mathrm{mg} / \mathrm{L}$, respectivamente, o que se refletiu na flutuação na eficiência de remoção, levando a um valor médio de $55,43 \%$. A partir do $28^{\circ}$ dia de operação, nota-se uma tendência de crescimento nos valores de remoção de DQO e estabilização do reator, aumentando sua eficiência de remoção de $\mathrm{DQO}$ e atingindo eficiência de $70 \%$.

A Figura 5 apresenta o comportamento da DQO (afluente e efluente), bem como a eficiência 
de remoção deste parâmetro ao longo do período operacional.

Embora a Resolução no 430/2011 do CONAMA (Brasil, 2011) não faça referência a DQO, a mesma cita como condição de lançamento de efluentes em corpos de água uma remoção mínima de $60 \%$ de $\mathrm{DBO}_{5,20}$. Como a eficiência de remoção de DQO estabilizou na faixa de $70 \%$, entende-se que a eficiência de remoção de matéria orgânica atende a legislação.

Pickbrenner (2002), em estudo de remoção de carbono e nutrientes, em RSB com ciclos de 12 e 6 horas, obteve eficiência de remoção de
DQO variando de $75 \%$ a $90 \%$, tratando efluente de UASB alimentado com esgoto sintético. No entanto, essa mesma autora, em instalação em escala piloto, operou dois RSB tratando efluente anaeróbio (50\%) e esgoto bruto $(50 \%)$ após tanque de fermentação, e a eficiência de remoção de DQO maior ou igual a $47 \%$ e $50 \%$ ocorreu somente em $50 \%$ do tempo da operação experimental.

Para entender como ocorria a degradação da DQO, no último ciclo operacional do RSB, foi realizado o perfil temporal de remoção de DQO apresentado na Figura 6.

Figura 5 - Comportamento da DQO ao logo do tempo de operação do RSB

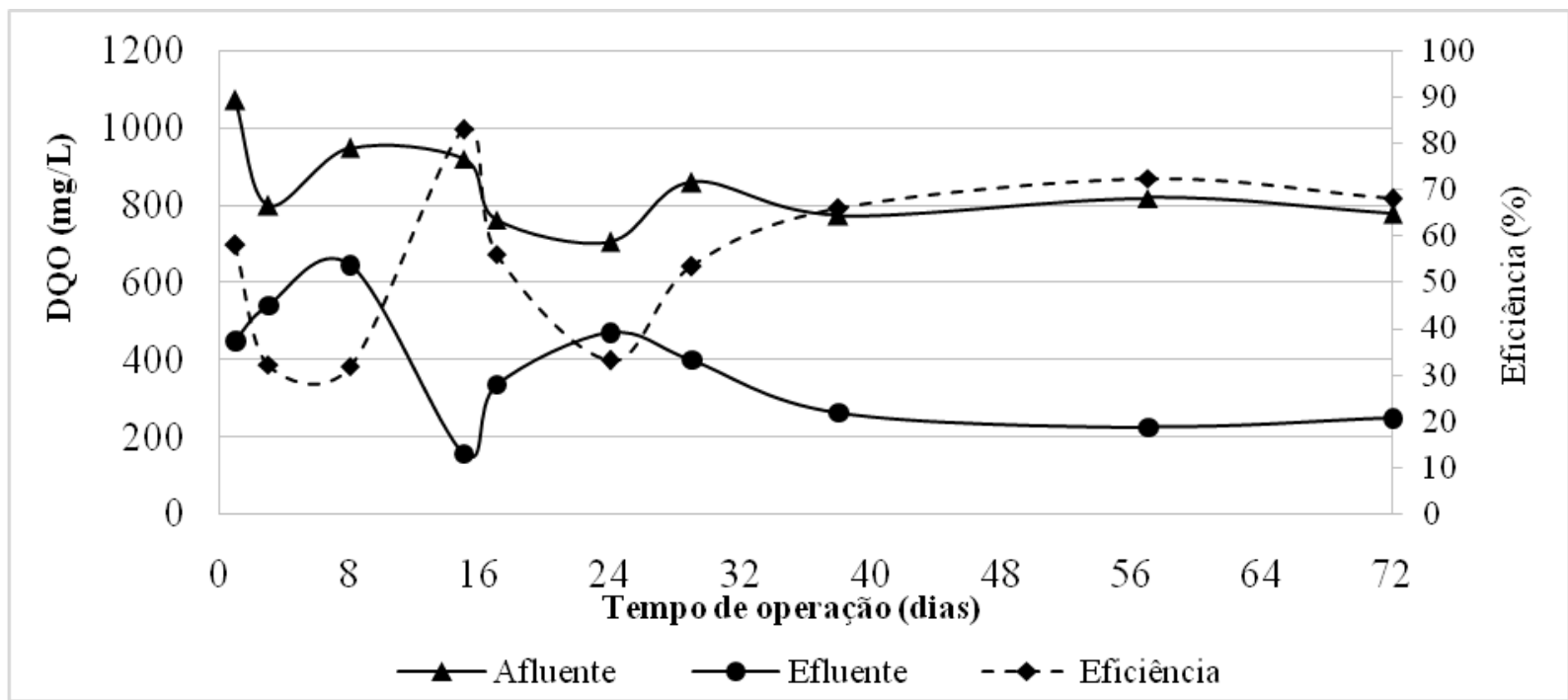

Fonte: Os Autores.

\section{Figura 6 - Perfil temporal da DQO no último ciclo do RSB}

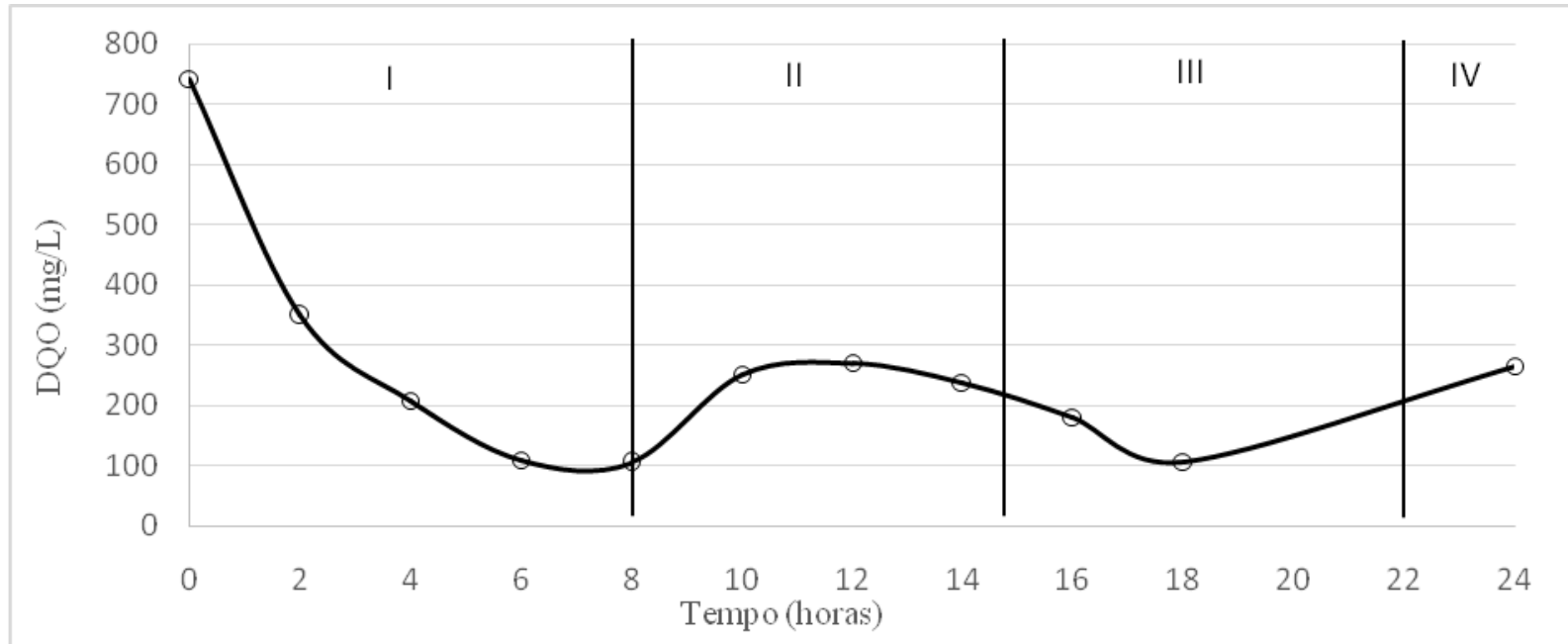

Legenda: I - Fase anaeróbia, II - Fase aeróbia, III - Fase anóxica, IV - Sedimentação/Descarte Fonte: Os Autores. 
Observando o gráfico da Figura 6, verificase que a maior fração da matéria orgânica era degrada na condição anaeróbia, nas seis primeiras horas de reação. Notou-se, também, um acréscimo nos valores da DQO entre o final da condição anaeróbia e o final do ciclo de reação, provavelmente, devido à suspensão dos sólidos acumulados no reator.

No final da operação, a concentração de sólidos verificada no lodo sedimentado era de $12.945 \mathrm{mg} / \mathrm{L}, \quad 5.066 \mathrm{mg} / \mathrm{L}$ e $7.879 \mathrm{mg} / \mathrm{L}$, respectivamente, para os sólidos totais, fixos e voláteis, cuja relação STV/SST era de 0,6, indicando que $60 \%$ dos sólidos presentes eram orgânicos, os quais foram considerados como biomassa. Isso resultava numa relação inicial DQO/SSV, na fase anaeróbia logo após a alimentação do RSB, de 0,32mgDQO/mgSSV.dia. $\mathrm{Na}$ fase aeróbia, quando o lodo estava em suspensão, a concentração de sólidos voláteis suspensos era de $2.195 \mathrm{mg} / \mathrm{L}$, o que resultava numa relação DQO/SSV de 0,17 $\mathrm{mg} . \mathrm{DQO} / \mathrm{mgSSV}$.dia. Vale salientar que não foi necessário fazer descarte programado de lodo, pois a altura da manta de lodo sedimentado permanecia na marca inicial de 2,0L de quando foi feita a inoculação do reator. Possivelmente, 0 crescimento da biomassa foi compensado por perdas eventuais durante os procedimentos de coleta de amostras para análises, 0 que contrabalanceava o volume de lodo no reator.

\section{CONCLUSÕES}

O Reator Sequencial em Batelada, operando com a fase de reação em períodos anaeróbio, aeróbio e anóxico, apresentou-se estável, tratando esgoto sanitário com DQO na faixa de $850 \mathrm{mg} / \mathrm{L}$ e nitrogênio de $54 \mathrm{mg} / \mathrm{L}$, com $265 \mathrm{mgDQO} / \mathrm{L}$ e $17 \mathrm{mgN}$-amoniacal e $4 \mathrm{mgN}$-nitrato em média no efluente. De acordo com a Resolução 430/2011 do CONAMA (Brasil, 2011), os resultados indicam a viabilidade do RBS com fases anaeróbia, aeróbia e anóxica e tempo de ciclo de $24 \mathrm{~h}$ para pequenos sistemas descentralizados de tratamento de esgotos sanitários no meio urbano.

O desempenho de remoção de matéria orgânica do reator foi crescente ao longo do tempo. A partir do $24^{\circ}$ dia operacional, a eficiência de remoção de DQO manteve-se na faixa de 69 $\%$.
O processo de nitrificação foi limitado em $72 \%$, possivelmente, em função da deficiência de oxigênio dissolvido. O processo de desnitrificação alcançou $75 \%$ em relação ao nitrogênio nitrificado, podendo ter sido limitado pela fonte de carbono disponível, provavelmente de não fácil biodegradação. A eficiência de remoção de nitrogênio atingiu no final da operação o valor de $42 \%$, sem adição de fonte externa de carbono.

\section{AGRADECIMENTOS}

A FAPEAL pela concessão de auxílio a pesquisa (edital 14/2016), e a CAPES pela concessão de bolsas de estudos.

\section{REFERÊNCIAS}

AKIN, B.S.; UGURLU, A. The effect of an anoxic zone on biological phosphorus release by a sequential batch reactor. Bioresource Technology, v. 94, n. 1, p. 1-7, 2004.

ALVES, C.F.; BORTOLUCI, C.B.C.; OLIVEIRA, E.P.; PIZZO, M.M.; RODRIGUEZ, R. P. (2018). Remoção biológica de nitrogênio em águas residuárias: uma revisão dos processos convencionais aos processos modernos. Revista lbero Americana de Ciências

Ambientais, v. 9, n. 3, p. 174-187, 2018. DOI: http://doi.org/10.6008/CBPC2179-6858.2018.003.0015

AMERICAN PUBLIC HEALTH ASSOCIATION (APHA); AMERICAN WATER WORKS ASSOCIATION (AWWA); WATER ENVIRONMENTAL FEDERATION (WEF).

Standard methods for the examination of water and wastewater. Washington, D.C.: American Public Health Association (APHA).

BUENO, R.F.; CAMPUS, F.; RIERA, M.M.R.; LENIS, C.; PIVELI, R.P. Simultaneous removal of organic material, nitrogen and phosphorus in a reactor in batches of mobile bed biofilm sequencing by the anaerobic-anoxic-oxic process. Engenharia Sanitária e Ambiental, Rio de Janeiro, v. 24, n. 4, jul./ago. 2019. Epub 06-Jun-2019. DOI: 10.1590/S141341522019125711.

BRASIL. Resolução CONAMA 430 de 13 de maio de 2011. Dispõe sobre as condições e padrões de lançamento de efluentes, complementa e altera a resolução no 357, de 17 de março de 2005, do Conselho Nacional do Meio Ambiente. Brasília: Diário Oficial da União de 16 de maio de 2011.

CALLADO, N.H.; FORESTI, E. Removal of organic carbon, nitrogen and phosphorus in sequential batch reactors integrating the aerobic/anaerobic processes. 
Water Science and Technology, v. 44, p. 263-270, 2001.

Lhttps://www.ncbi.nlm.nih.gov/pubmed/11575092.

CHEN, H.; LIU, Y.; NI, B.J.; WANG, Q.; WANG, D.; ZHANG, C.; LI, X.-M.; ZENG, G. Full-scale evaluation of aerobic/extended-idle regime inducing biological phosphorus removal and its integration with intermittent sand filter to treat domestic sewage discharged from highway rest area. Biochemical Engineering Journal, v. 113, p. 114-122, 2016. http://dx.doi.org/10.1016/j.bej.2016.06.002.

CHIU, Y.-C.; LEE, L.-L.; CHANG, C.-N.; CHAO, A.C. Control of carbon and ammonium ratio for simultaneous nitrification and denitrification in a sequencing batch bioreactor. International Biodeterioration \& Biodegradation, v. 59, n. 1, p. 1-7, 2007.

DAMIANOVIC, M.H.R.Z.; SANTOS, C.E.D.; MARTÍN, M.A.M.; FDZ-POLANCO, M.; FORESTI, E.; FDZPOLANCO, F.; GARCÍA-ENCINA, P.A. Specific activity bioassays as tools to evaluate combined nitrogen and organic matter removal in SND systems.

Environmental Engineering Science, v. 35, n. 9, 2018. Published Online: 1 Sep 2018.

https://doi.org/10.1089/ees.2017.0418

DILALLO, R.; ALBERTSON, O.E. Volatile acids by direct titration. Journal Water Pollution Control Federation, v. 33, n. 4, p. 356-365, 1961.

FOCO, M.L.R.; NOUR, E.A.A. Desempenho de sistema combinado anaeróbio-aeróbio na remoção de nitrogênio no tratamento de esgoto sanitário. Ciências Exatas e Tecnologia, Londrina, v. 35, n. 2, p. 131-138, 2014.

FONTENOT, Q.; BONVILLAIN, C.; KILGEN, M.; BOOPATHY. R. Effects of temperature, salinity, and carbon: nitrogen ratio on sequencing batch reactor treating shrimp aquaculture wastewater. Bioresource Technology, n. 98, p. 1700-1703, 2007.

GONZALEZ-GIL, G.; SOUGRAT, R.; BEHZAD, A.R.; LENS, P.N.L.; SAIKALY, P.E. Microbial community composition and ultrastructure of granules from a fullscale anammox reactor. Microbial Ecology, v. 70, n. 1, p. 118-131, 2015.

HU, X.; XIE, L.; SHIM, H.; ZHANG, S.; YANG, D. Biological nutrient removal in a full scale anoxic/anaerobic/aerobic/pre-anoxic-MBR plant for low $\mathrm{C} / \mathrm{N}$ ratio municipal wastewater treatment. Chinese Journal of Chemistry Engineering, v. 22, n. 4, p. 447454, 2014. https://doi.org/10.1016/S10049541(14)60064-1.
LI, X.-M.; CHEN, H.-Bo; YANG, Qi Y.; WANG, D.-Bo; LUO, K.; ZENG, G.-M. Biological nutrient removal in a sequencing batch reactor operated as oxic/anoxic/extended-idle regime. Chemosphere, v.105, p. 75-81, 2014.

LI, Y.Z.; HE, Y.L.; OHANDJA, D.G.; JI, J.F.; ZHOU, T. Simultaneous nitrification-denitrification achieved by an innovative internal-loop airlift MBR: Comparative study. Bioresource Technology, v. 99, n. 13, p. 5867-5872, 2008.

MACKERTH, F.J.H.; HERONS, J.; TALLING, J.F. Water analysis: some revised methods for limnologists. Scientific publication, n. 36. London: Freshwater Biological Association, 1978.

MENG, Q.; YANG, F.; LIU, L.; MENG, F. Effects of $\mathrm{COD} / \mathrm{N}$ ratio and $\mathrm{DO}$ concentration on simultaneous nitrification and denitrification in an airlift internal circulation membrane bioreactor. Journal of Environmental Sciences, v. 20, p. 933-939, 2008.

METCALF, L.; EDDY, H.P. Tratamento de efluentes e recuperação de recursos. 5. ed. Nova lorque: Ed. AMGH, 2008. p. 2016.

PEREIRA, T.D.S.; SANTOS, C.E.D.; LU, Xi; AL-HAZMI, H.E.; MAJTACZ, J.; PIRES, E.C.; DAMIANOVIC, M.H.R.Z.; MAKINIA, J. Effect of operating conditions on $\mathrm{N}_{2} \mathrm{O}$ production in ananammox sequencing batch reactor containing granular Sludge. WS\&T, v. 80, n. 1, 2019. doi: 10.2166/wst.2019.237.

PICKBRENNER, K. Uso de reator seqüencial em batelada (rsb) para pós-tratamento de efluente de reator anaeróbio. 2002. Dissertação (Mestrado) Instituto de Pesquisas Hidráulicas, Universidade Federal do Rio Grande do Sul, Porto Alegre/RS. 2002.

RIPLEY, L.E; BOYLE, W.C; CONVERSE, J.C. Improved alkalimetric monitoring for anaerobic digestion of high- strength wastes. Journal Water Pollution Control Federation, Alexandria, v. 58, n. 5, p. 406-411, 1986.

SANTOS, C.E.D.; MOURA, R. B.; DAMIANOVIC, M.H.R.Z.; FORESTI, E. Influence of COD/N ratio and carbon source on nitrogen removal in a structured-bed reactor subjected to recirculation and intermittent aeration (SBRRIA). Journal of Environmental Management JCR , v. 166, p. 519-524, 2016.

SILVA, W. F. da. Análise da vulnerabilidade das águas subterrâneas à contaminação na região metropolitana de Maceió. 2013. Dissertação (Mestrado em Recursos Hídricos e Saneamento) - 
Centro de Tecnologia, Universidade Federal de Alagoas, Maceió/AL. 2013.

TORRES, P. Desempenho de um reator anaeróbio de manta de lodo (UASB) de bancada no tratamento de substrato sintético simulando esgotos

sanitários. 1992. Dissertação (Mestrado) - Escola de Engenharia de São Carlos, Universidade de São Paulo, São Carlos/SC. 1992.

TSUNEDA, S.; OHNO, T.; SOEJIMA, K.; HIRATA, A. Simultaneous nitrogen and phosphorus removal using denitrifying phosphate-accumulating organisms in a sequencing batch reactor. Biochemical Engineering Journal, v. 27, p. 191-196, 2006.
XU, X.; LIU, G.; ZHU, L. Enhanced denitrifying phosphorous removal in a novel anaerobic/aerobic/anoxic (AOA) process with the diversion of internal carbon source. Bioresource Technology, v. 102, p. 10340-10345, 2013.

ZOPPAS, F.M.; BERNARDES, A.M.; MENEGUZZI, A. Parâmetros operacionais na remoção biológica de nitrogênio de águas por nitrificação e desnitrificação simultânea. Engenharia Sanitária e Ambiental, v. 21 n. 1, p. 29-42, jan./mar. 2016. DOI: 10.1590/S141341520201600100134682. 